\section{Cahiers de Narratologie}

Analyse et théorie narratives

$10.1 \mid 2001$

La voix narrative

\title{
Tradition orale et instance narratrice dans En attendant le vote des bêtes sauvages de Ahmadou Kourouma
}

\section{Clément Animan}

\section{(2) OpenEdition}

Journals

Electronic version

URL: http://journals.openedition.org/narratologie/6919

DOI: 10.4000/narratologie.6919

ISSN: 1765-307X

Publisher

LIRCES

\section{Printed version}

Date of publication: 1 January 2001

Number of pages: 89-101

ISBN: 2914561032

ISSN: 0993-8516

Electronic reference

Clément Animan, "Tradition orale et instance narratrice dans En attendant le vote des bêtes sauvages de Ahmadou Kourouma", Cahiers de Narratologie [Online], 10.1 | 2001, Online since 16 October 2014 , connection on 23 February 2021. URL: http://journals.openedition.org/narratologie/6919 ; DOI: https:// doi.org/10.4000/narratologie.6919 


\title{
TRADITION ORALE ET INSTANCE NARRATRICE DANS EN ATTENDANT LE VOTE DES BETES SAUVAGES DE AHMADOU KOUROUMA.
}

\author{
Clément ANIMAN
}

Institut international de Sociocritique/Montpellier Université d’Alcalá-Madrid/Espagne

A Ézéchiel, le kouroumiste A Ali, le «Babalawo », pour nos veillées madrilènes sous l'arbre à palabre

Mystérieusement et brusquement un tourbillon de vent se déclenche, naît au milieu du jardin de la Résidence. Le tourbillon soulève feuilles et poussière, parcourt le jardin de la Résidence d'ouest en est et poursuit sa folle course dans la cour voisine, dans l'enceinte de l'Ambassade des USA. Koyaga comprend tout de suite que le grand initié Fricassa Santos s'est transformé en vent pour se réfugier dans l'ambassade. Du balcon du premier étage Koyaga suit le mouvement du tourbillon qui, brusquement, auprès d'une vieille voiture garée dans le jardin, s'évanouit, se dissipe. Le grand initié Fricassa Santos sort du vent et se découvre, déguisé en jardinier.

Les non-initiés, par ignorance, douteront de cette version des faits. Ils prétendront qu'un passage souterrain existait entre la résidence du président et l'enceinte de l'ambassade. Le président aurait, déguisé, emprunté ce passage dans l'obscurité et se serait recroquevillé sur la banquette arrière de la voiture toute la nuit. Il serait sorti de la voiture Buik quand les grilles de l'ambassade se sont ouvertes. C'est évidemment une explication enfantine de Blanc qui a besoin de rationalité pour comprendre.

Dès que le président apparaît en entier, en totalité, les tirailleurs, sans respect des conventions diplomatiques, pénètrent dans l'enceinte de l'ambassade, s'emparent du 
Président, le rudoient, le bousculent, l'entraînent dans la rue.

Dès que le Président passe la grille et se trouve hors de l'enceinte, un tirailleur fait feu et, curieusement, manque le Président. Il ne l'a pas manqué (on ne rate pas à bout portant), mais les objets de métal ne pénètrent pas dans la chair d'un grand initié. Les soldats le savaient; on le leur avait plusieurs fois répété. Ils sont décontenancés, dépassés, terrorisés. Ils jettent leurs armes et détalent. Le président seul dans la rue se dirige tranquillement vers l'ambassade. Koyaga accourt et, avant que le Président atteigne la grille, il décoche de son arc une flèche de bambou agencée au bout d'un ergot de coq empoisonné. Les devins avaient révélé au chasseur que seule une flèche dotée d'un ergot de coq empoisonné pouvait annihiler le blindage magique du super-initié qu'était le Président, pouvait rendre sa peau et sa chair pénétrables par du métal. La flèche se fixe dans l'épaule droite. Le président saigne, chancelle et s'assied dans le sable. Koyaga fait signe aux soldats. Ils comprennent et reviennent, récupèrent leurs armes et les déchargent sur le malheureux Président. le grand initié Fricassa Santos s'écroule et râle. Un soldat l'achève d'une rafale. Deux autres se penchent sur le corps. Ils déboutonnent le Président, l'émasculent, enfoncent le sexe ensanglanté entre les dents. C'est l'émasculation rituelle. Toute vie humaine porte une force immanente. Une force immanente qui venge le mort en s'attaquant à son tueur. Le tueur peut neutraliser la force immanente en émasculant la victime.

En attendant le vote des bêtes sauvages, Veillée II, pp. 93-94.

Ces pages, tirées de En attendant le vote des bêtes sauvages de Ahmadou Kourouma ${ }^{1}$, sont relatives à la dernière séquence de l'épisode sur l'assassinat du président Fricassa Santos par un quator de conjurés ayant à leur tête le sergent Koyaga, maître chasseur, puis futur président-dictateur de la République du Golf, et constituent le texte narratif de base à notre étude. Il convient, peut-être, de faire remarquer, par

${ }^{1}$ Paris, Seuil, 1998. 
ailleurs, qu'au regard du thème de En attendant le vote des bêtes sauvages, il s'agit de l'histoire de la dictature de Koyaga et de ses avatars, les mensonges, les crimes et les assassinats. Quant au récit qui assure cette histoire, il est purificatoire, à en croire ce qu'annonce le griot Bingo :

Je dirai le récit purificatoire de votre vie de maître chasseur et de dictateur. Le récit purificatoire est appelé en Malinké un donsomana. C'est une geste. Il est dit par un sora accompagné par un cordoua. Un cordoua est un initié en phase en phase purificatoire, en phase cathartique. Tiécoura est un cordoua et comme tout cordoua il fait le bouffon, le pitre, le fou. Il se permet tout et il n'y a rien qu'on ne lui pardonne pas ${ }^{2}$

Nous voyons poindre déjà à travers les gens de la parole - le sora et le cordoua, griot et disciple de griot, respectivement - la tradition orale (je dirai le récit), comme texte narratif majeur de la geste que constitue En attendant le vote des bêtes sauvages. Mais encore : le griot apparaît comme un hagiographe, le dépositoire de l'histoire de Koyaga. Celle-ci étant une geste, cela nous conduit, nécessairement à penser que :

1. il s'agit bien là d'un récit mythique au sens où la geste met traditionnellement en jeu les exploits originels des héros et n'est pas susceptible

2. d'être traversée par un contre-récit, vu son caractère sacré et exemplaire.

Seulement voilà, l'incipit nous situe mieux sur les personnages du sora et du cordoua, à travers les prétentions du premier :

Moi, Bingo, je suis le sora; je louange, chante, et joue de la cora. Un sora est un chantre, un aède qui dit les exploits des chasseurs et encense les héros chasseurs. Retenez mon nom de Bingo, je suis le griot musicien de la confrérie des chasseurs.

2 Ibid., p. 10. 
L'homme à ma droite, le saltimbanque accoutré dans ce costume effarant, avec la flûte, s'appelle Tiécoura. Tiécoura est mon répondeur.

Le répondeur joue de la flûte, gigote, danse. Brusquement, s'arrête et interpelle le président Koyaga.

- Président, général et dictateur Koyaga, nous chanterons et danserons votre donsomana en cinq veillées. Nous dirons la vérité. La vérité sur votre dictature. La vérité sur vos parents, vos collaborateurs. Toute la vérité sur vos saloperies, vos conneries; nous dénoncerons vos mensonges, vos nombreux crimes et assassinats...

On ne pourrait s'empêcher de dire que Tiécoura, le cordoua, répondeur du griot musicien des chasseurs n'est pas, contre toute attente d'ailleurs, une simple caisse de résonance. $\mathrm{Ce}$, d'autant plus qu'au récit annoncé de louanges de Bingo s'oppose son contre-récit vaticiné, aussi, comme celui de vérités. $\mathrm{Ce}$, à travers une microsémiotique propre au réquisitoire judiciaire (la vérité... toute la vérité). Edmond Cros à qui nous empruntons la notion de microsémiotique la définit comme le discours spécifique des groupes ou catégories sociales ${ }^{3}$.

Le conflit pouvoir de dire / pouvoir de contredire se trouve reproduit dans le texte à travers la tension mensonges /vérités ou encore celle de mythe / réalité. Ce qui nous autorise à dire que c'est l'opposition pouvoir de dire Ipouvoir de contredire, présente dès le début du texte, qui programme la narration. Dès lors, cela revient à poser, à la suite d'Edmond Cros, l'hypothèse que l'instance narratrice est le produit du texte narratif.

En privilégiant le concept d'instance narratrice, telle que proposée par Cros, nous cessons de faire du narrateur, le dépositaire exclusif de la narration, nous amenant, de ce fait, à voir dans des voix contradictoires, les tenants d'un récit

${ }^{3}$ Genèse socio-idéologique des formes, Montpellier, CERS, 1998, p. 32 . 
produit par le texte narratif. A partir de là, nous voulons montrer que, dans la mise en rapport entre la tradition orale et l'instance narratrice, le récit est traversé par des voix en conflit qui mettent en crise une Histoire officielle, identifiée à la geste, donc, sacrée et, partant, totalitaire.

Avant de revenir à l'extrait qui nous occupe, il nous semble d'une extrême importance de donner à connaître d'autres extraits de l'incipit pour mieux poser la question du narrateur :

Votre nom: Koyaga! Votre totem : faucon. Vous êtes soldat et président. Vous êtes chasseur! Vous resterez avec Ramsès II et Soundjata l'un des trois plus grands chasseurs de l'humanité. C'est bientôt la nuit. Vous avez convoqué les sept plus prestigieux maîtres parmi la foule des chasseurs accourus. Vous Koyaga, trônez, dans le fauteuil au centre du cercle. maclédio, votre ministre de l'Orientation, est installé à votre droite. Retenez mon nom de Bingo, je suis le griot musicien de la confrérie des chasseurs ${ }^{4}$.

Il y a là un narrataire identifiable qui est Koyaga et il y a aussi une instance narratrice dont le mode de récit est celui de la focalisation externe -narrateur extradiégétique- dans la mesure où Bingo s'identifie à un témoin des faits des chasseurs (je suis... de la confrérie des chasseurs) et en est mandaté -mais bien de l'extérieur- pour conter les faits.

Rappelons, pour notre propre éclairage, que tous les narrataires, c'est-à-dire, Koyaga, les chasseurs, Maclédio et le répondeur (Tiécoura) sont des initiés (pp. 10,61) et le récit de la vie de Koyaga se déroule la nuit - il se déroulera tout le long du roman, en six veillées. Mis à écouter - ou à lire - le récit, le lecteur est appelé à être, soit, un voyeur : dans ce cas, ce n'est pas un initié, et il cherche à franchir les limites du mystère. Il veut, pour tout dire, le transgresser ; soit, le lecteur est un initié et, alors, il comprend l'irruption de l'extraordinaire dans la réalité quotidienne - mais comment le peut-il quand, dans ces conditions, il est convoqué par la métaphy-

${ }^{4}$ En attendant le vote des bêtes sauvages, op. cit., p. 9. 
sique dans un monde dominé par la logique rationaliste ? Autant le dire : le lecteur ne restera, tout au long de ce face à face en absence avec le texte, qu'un non-initié qui essaiera de déchiffrer le moindre des mystères par la médiation textuelle du répondeur Tiécoura.

C'est Sory Camara qui semble nous donner les raisons du mystère qui entoure la parole dans l'Afrique précoloniale et, principalement, chez les Malinkés :

Il en résulte que le Malinké tait généralement tout ce qu'il n'est pas nécessaire de dire. Lorsqu'il est obligé de parler, car il faut bien parler, il aime à s'exprimer par énigmes: d'où l'importance des proverbes, même dans les conversations les plus banales. Cela permet de parler avec cette sorte de pudeur qui laisse aux choses leur dimension mystérieuse ${ }^{5}$.

La parole, pour le Malinké est énigme et mystère, autrement elle devient subversion, car comme le confirme Sory camara, la parole, pour le Malinké «fait sortir le fond des choses. Elle en révèle le sens, elle exhume le dessous. C'est là précisément, le désordre, la rupture d'harmonie ${ }^{6}$

On fera remarquer qu'une des catégories sociales du malinké, c'est-à-dire, le griot, a investi En attendant le vote des bêtes sauvages et s'adonne - comme narrateur, dont les paroles sont narrativisées, pour parler comme Genette ${ }^{7}-$ aux énigmes à travers la récurrence des proverbes : ce sont vingtquatre proverbes qui fonctionnent comme autant de paratextes des vingt-quatre chapitres du roman.

$\mathrm{Si}$, à présent, nous revenions au texte proposé pour notre analyse, nous nous rendrions vite compte que le narrateur, use, comme nous le disions à propos de l'incipit, d'une voix objective, puisque le recours à la troisième personne du

${ }^{5}$ Gens de la parole, Kartala, Paris, 1992, p. 255.

${ }^{6}$ Ibid.

7 « Récit de paroles» en Figures III, op. cit., p. 190. 
singulier est récurrent. Mais, aussitôt après, nous assistons à un glissement de la focalisation externe à une focalisation zéro, à partir du moment où le narrateur par l'incursion dans l'intériorité de Koyaga (Koyaga comprend), semble tout savoir, même des pensées les plus intimes. Quant au récit luimême, il est mystérieux, énigmatique à en juger par les signes mystérieusement et initié. Le personnage principal de l'épisode, Fricassa Santos, semble être un être fabuleux, étant donné qu'il y a rupture d'avec le cours normal des événements. Autant dire : il y a une irruption de l'inhabituel dans la monotonie des représentations traditionnelles de la réalité, irruption inhabituelle marquée par la répétition de brusquement. Par ailleurs, on apprend aussi que « Fricassa Santos sort du vent ».

Devant l'incrédulité implicite de l'hypothétique narrataire ou lecteur (les non-initiés), intervient celui que Madeleine Borgomano appelle le narrateur régisseur ${ }^{8}$. Celui qui, selon elle, "régit l'ensemble du discours du roman et, domine, ou organise, d'une certaine façon, toutes les autres voix $"{ }^{9}$. Pour notre part, nous croyons que le narrateur régisseur correspond, ici, au narrateur omniscient, étant donné qu'à travers la prolepse (douteront), il anticipe les événements et se pose en maître du temps et en détenteur exclusif de la vérité. Cependant, il paraît impartial - ce qui entre en contradiction avec l'objectivité que confère la narration à la troisième personne - puisque l'ignorance dont il parle semble s'assimiler au mensonge, dans l'allusion à l'explication enfantine et rend impossible l'émergence d'un narrateuropposant. Car, le narrateur régisseur élude la contradiction en appelant au maintien de l'ordre sacré du mystère qui s'oppose, dans son discours, à l'élucidation latente dans le signe rationalité.

Cette même tension vérité exclusive /contradiction implicite est présente dans la séquence relative à comment

${ }^{8}$ Ahmadou Kourouma. Le "guerrier griot», Paris, L'Harmattan 1998, p. 162.

${ }^{9}$ Ibid. 
Koyaga, encore simple ancien combattant d'Indochine échappe aux hommes venus l'assassiner pour le compte du président Fricassa Santos contraint de l'éliminer s'il veut se soustraire à la prédestination d'être tué par Koyaga :

Koyaga récite une des prières que le marabout lui a apprises : il se transforme en un coq blanc. Le Haoussa voit le coq sous son banc; il le croit échappé d'un de ses paniers. Vigoureusement le marchand se saisit du coq, l'enfouit et l'enferme dans le panier, sort de la gare devant les policiers en civil avec toute sa volaille. La version des faits qui veut que Koyaga soit descendu du train travesti en marchand haoussa de poulets n'est pas crédible; il y avait trop de policiers perspicaces à l'arrivée du train. Le chasseur n'aurait pas pu leur échapper s'il était passé sous un simple déguisement ${ }^{10}$

Ici, le narrateur-opposant est contemporain au narrateur régisseur, car le temps utilisé est le présent de l'indicatif (la version qui veut que). Mais une fois encore, le narrateur régisseur censure la voix discordante, en mettant en cause sa crédibilité (n'est pas crédible) : toute parole qui n'énonce pas des faits surnaturels semble, donc, être frappée d'interdit. De sorte que, dans la majorité des cas, la plupart des narrataires interpellés par le narrateur régisseur sont des voix multiples qui participent, à certains moments, à la narration. Mais, en général, ce sont des voix que nous nommerons narrateursadjuvants, c'est-à-dire des voix qui assurent la continuité ou se font l'écho du récit du narrateur régisseur. Voyons comment, à travers quelques exemples :

1. Au bureau du capitaine, on vous détailla de nombreux avantages attribués aux réengagés pour l'Algérie et vous paya comptant le pécule d'ancien combattant d'Indochine. plus de cent mille francs CFA! ... Trop riche, vous avez tout négligé et oublié, et tout de suite du bureau, vous avez couru pour vous procurer un fusil, le mettre en bandoulière et attraper le premier taxi-brousse

${ }^{10}$ Ibid., p. 84. 
Tradition orale et instance narrative dans

En attendant le vote des bêtes sauvages de A. Kourouma

en partance pour les montagnes, complète le répondeur"l.

2. Boum! le coup de Koyaga partit et la panthère s'affala. Le monstre gorgé de sang venait de passer de vie à trépas.

- En plantant la fin de la bête (sa queue) dans son commencement, tous les nyamas étaient condamnés à rester, à continuer à tourner en circuit fermé dans les restes de la bête, explique Maclédio ${ }^{12}$.

Les narrataires, Tiécoura et Maclédio, en complétant le récit, ou encore, en en donnant des précisions ou des explications, nous donnent à écouter - ou à lire - un récit collectif où eux-mêmes finissent par être des narrateurs dont le mode de récit est la focalisation externe, se faisant, ainsi, porteurs d'une vision objective du récit sur le parcours initiatique de Koyaga (=le combat épique contre le monstre ou la Bête).

En tout cas, Sory Camara indique, dans Gens de la parole, qu'hormis la littérature orale d'élite assumée de bout en bout par les griots, il existe aussi une littérature populaire - orale, également-, sans auteur, dont chacun se fait à son tour l'interprète :

Leur narration même indique leur nature collective: chaque phrase du narrateur est ponctuée par l'intervention du námúnámúlá (celui qui dit námú, " c'est cela ») ou répondant. Le récit se termine par une formule qui oblige le narrateur à céder la parole à un autre ${ }^{13}$.

On conviendra que le récit collectif, dans En attendant le vote des bêtes sauvages, est en jeu quand les événements sont à un temps prétérit, comme une sorte de récit transmis de génération en génération. Par contre, quand le temps de la narration est au présent, donc, d'actualité, avec les moyens

${ }^{11}$ En attendant le vote..., op. cit., p. 64.

12 Ibid., p. 66.

${ }^{13}$ Gens de la parole, Paris, Karthala, 1992, p. 263. 
modernes d'information - comme dans la séquence où l'on découvre sous l'œil vigilant de la presse écrite, de la radio et de la télévision, des documents d'une supposée conspiration communiste contre Koyaga ${ }^{14}$-, la parole devient un puissant facteur de contre-pouvoir. Tâche qui, en règle générale, semble être assurée par Tiécoura, disciple et répondeur de Bingo, le maître griot :

1. Le président ne pouvait pas, pendant ses heures habituelles de réception, de bureau, y consacrer le temps nécessaire sans être contraint à toutes autres obligations de chef d'État, de dictateur, de père de la nation.

- Et aussi de gros coureur de femmes d'autrui, les femmes des citoyens. Ajoute malicieusement le répondeur ${ }^{15}$.

2. Les prisonniers politiques comprennent qu'ils sont abandonnés par les hommes, les dieux et les religions. Ils paniquent et se suicident.

- Dans leur rage suicidaire et sanguinaire, ils s'amputent des sexes avant de passer à l'action, ironise le répondeur ${ }^{16}$.

On voit, ici, que le répondeur, parce que disciple de griot et initié, est redistribué, par rapport à son rôle traditionnel de répondeur, donc, de caisse de résonance du maître. Il y a déjà dans le premier exemple, les signes d'une parole, d'une voix, d'un narrateur désormais subversif qui met en crise l'authenticité de l'Histoire officielle.

La référence à l'activité sexuelle de Koyaga est une exhibition de l'intimité du président-dictateur, provoquant une transgression de l'ordre, puisque selon Sory Camara, il existe chez le griot la parole injurieuse considérée comme «une arrogance et un désordre »17 lorsqu'elle touche à l'intimité. Le narrateur Tiécoura tourne, surtout, en dérision

${ }^{14}$ En attendant le vote des bêtes sauvages, op. cit., p. 270.

15 Ibid., p. 265.

16 Ibid., p. 275.

${ }^{17}$ Gens de la parole, op. cit., p. 256. 
(malicieusement) l'image préconstruite du père, présente dans la périphrase, père de la nation. En considérant la notion de père de la nation comme archétype du père, on se rend bien à l'évidence que les valeurs morales élémentaires sont bafouées par Koyaga : c'est un père adultérin (femmes d'autrui) et incestueux, l'allusion aux femmes pouvant être vue, au regard de la notion du père, comme une référence à l'archétype de la progéniture féminine.

Dans le deuxième exemple, la vérité absolue du narrateur omniscient (une fois de plus, il sait tout, puisqu'il arrive à sonder l'intérieur des prisonniers au moment de leur supposé suicide volontaire : les prisonniers politiques comprennent) est renversée par la parole de Tiécoura. Celui-ci a recours à l'anti-phrase pour montrer l'absurdité de la thèse officielle du suicide car l'on ne peut parler de rage suicidaire et sanguinaire pour décrire l'état d'âme de personnes qui, dans les pages antérieures, ont eu recours aux organisations occidentales des droits de l'homme pour les défendre ${ }^{18}$.

En somme, sous le mode de la focalisation externe et la focalisation zéro (narrateur omniscient), le récit sur la vie du dictateur-président Koyaga, s'est avéré être ce que Genette appelle le récit de paroles, puisque le narrateur régisseur n'est rien d'autre qu'un des gens de la parole, c'est-à-dire un griot. Une parole totalitaire, sacrée et qui, apparemment, communie avec la tradition orale. Malgré tout, la parole narrativisée se trouve, en d'autres endroits, plus subversive parce qu'informée, croyons-nous - ou du moins le roman, comme nous le verrons par la suite, nous permet de le dire - par des moyens modernes de contrôle de l'information.

Dans les dernières pages de En attendant le vote des bêtes sauvages, on se rend bien compte que la mise à jour de la parole subversive est signe du passage de la société traditionnelle mystérieuse et occulte - dont les hauts faits peuvent être seulement connus des initiés - à une société moderne où règne l'écriture :

${ }^{18}$ En attendant le vote des bêtes sauvages, op. cit., p. 274. 
Le nègre est un peuple sans écriture. Ce sont les colonisateurs, les curés et les marabouts qui l'ont alphabétisé. Ses maîtres lui ont inculqué le respect de l'écrit; le papier est un fétiche, une croyance. Une croyance qui, comme les textes des livres sacrés ou les ordres du colonisateur blanc, dépasse l'entendement du Nègre, ne se vérifie pas, ne se contredit pas ${ }^{19}$.

En excommuniant l'écriture, le narrateur régisseur, fait de la parole, surtout de la parole d'élite (Sory Camara) ${ }^{20}$ - celle du griot, par exemple - l'unique mode de savoir en Afrique.

Pourtant, pour faire vite et conclure, l'on voit bien que Tiécoura, le griot répondeur et l'écriture - médiatisée dans le livre de Kourouma, par les moyens d'information - en conflit avec la tradition orale, sont là pour rappeler que ce sont bien des voix plurielles et surtout contradictoires qui traversent l'instance narratrice, faisant d'elle, non l'énonciatrice d'une Histoire officielle, mais plutôt, le dépositaire de conflits (de "vérités ») qui informent l'histoire du roman, mais encore, l'Histoire de l'Afrique.

${ }^{19}$ Ibid., p. 329.

${ }^{20}$ Gens de la parole, op. cit., p. 263. 


\section{OUVRAGES CONSULTÉS}

BORgomano (Madeleine) : Ahmadou Kourouma. Le " guerrier griot ", Paris, L'Harmattan, 1998.

CAMARA (Sory) : Gens de la parole, Paris, Karthala, 1992.

CROS (Edmond) : Genèse socio-idéologique des formes, Montpellier, CERS, 1998.

GENETTE (Gérard) : Figures III, Paris, Seuil, 1972.

KOUROUMA (Ahmadou) : En attendant le vote des bêtes sauvages, Paris, Seuil, 1998. 